\title{
Working Mechanism and Its Application of Mechanical Principle Decomposition Symmetry
}

\author{
Zhimin Qiu ${ }^{1, \text { a }}$, Hui Chen ${ }^{2}$ \\ ${ }^{1,2}$ Nanchang Institute of Science and Technology, 330108 \\ aqiuzhiminjx@126.com
}

Keywords: principle symmetry principle decomposition symmetry design principle

\begin{abstract}
In the symmetry of mechanical system, we explored and made researches of related applications and the roles of mechanical principle decomposition symmetry, applied it to the real innovative product design. We established principle of space-time symmetry and principle of effect symmetry in the mechanical system which would consist of the symmetric system of analytic theory. Principle decomposition symmetry this thesis studied can be further divided into heterogeneous principle decomposition symmetry and homogeneous principle decomposition symmetry. Based on principle decomposition symmetry's working mechanism and function, we can get a relationship system, for example, from product's performance requirements to the advantages and disadvantages of the products based on the principle decomposition, and then form the principle for design of principle decomposition.
\end{abstract}

Symmetry exists widely in the natural or man-made products from articles for daily use to machinery products, especially in physics, the study of symmetry has got very rich achievements. The study of symmetry in the mechanical system is also very popular. In fact, the so-called mechanical symmetry must be symmetrical or repetitive as for the structure, principle and function in various mechanical systems.

There are three elements in mechanical symmetry, the main body, component and benchmark. The mechanical system principle decomposition symmetry enhances the principle of product innovation process. Based on the principle decomposition symmetry, more innovative products can be invented, the inherent function and performance of the machinery can be improved.

\section{Introduction of Mechanical Principle Symmetry}

What is principle symmetry, to put it simple, multiple types of principle or the combinations of them performing the same function. The core of principle symmetry is the principles. In order to achieve the same function, multiple of the these principles combine. Among these combinations, symmetrical benchmark achieves the same function for these combinations. Based on the symmetry of various disciplines, as well as the mechanical symmetry, the mechanical symmetry can be better summarized and concluded. In the mechanical system, symmetry almost appears in all kinds of mechanical system in the mechanical system at the same time and develops with the development of mechanical system. It ranges from the development of initial geometric multi-level product and product function principle etc.. Small mechanical standard parts, mechanical mechanism and system, such as specific geometric shapes, abstract function principle, from static to dynamic symmetry, symmetry exists in almost every aspect of mechanical system, and has a significant influence on the mechanical system. The concept of Symmetry is the acquisition of knowledge and the significance of research for applied mechanical effects, which is constructive to understand symmetry in mechanical system comprehensively and clearly, and further study the existence, development and the applicable of law of mechanical system. The study of the case system of mechanical symmetry applies theory of knowledge discovery, knowledge discovery methods and techniques to mechanical symmetry case. The knowledge of machinery design which comprehensively discovers machinery design knowledge will greatly expand the knowledge space. At the same time, the establishment of the method for knowledge discovery of mechanical symmetry provides a reference for the 
discovery of the knowledge for engineering design. The following is the structure of mechanical principle symmetry.

\section{Classifications of the Principle Composition Symmetry}

The classification of the principle decomposition of symmetry can be classified into heterogeneous, homogeneous, series, parallel and series-parallel principle decomposition symmetry according to its mechanism of function and arrangement.

First, heterogeneous effect decomposition symmetry. An effective decomposition effect principle can realize the same objective function. It can be further divided into series parallel decomposition according to the connection between the coupling effect. "providing magnetic field" is treated as symmetric decomposition. Compared with the passive magnetic bearings applying the "permanent magnet magnetic", hybrid magnetic bearing using "magnetic induction" and permanent magnet has heterogeneous effect parallel decomposition symmetry.

Second, Heterogeneous movement decomposition symmetry. In principle, the function is transformed movement into a variety of functions in movement, and finally achieve the same objective function together. For example, adjusting the position of the screen for the reference of symmetry and can only compare along the horizontal axis on the screen. Most of the notebook computers, the screens can rotate different symmetry decomposition respectively along the horizontal axis and vertical axis of rotary laptop.

Thirdly, principle series decomposition symmetry. Many sub-principles decomposed by symmetry successively decompose according to a certain sequence. For example, compared with the single-stage rocket, rocket with several stages in multiple rocket ignition has the same symmetry of series decomposition.

Fourth, principle series and parallel decomposition symmetry. Multiple sub-principles get or do not have the difference between each other at the same time can be divided into the following modes according to the different movements: (1) the parallel decomposition: multiple parallel sub-principles decompose at the same time. For example, compared with single rotor system, a helicopter rotor of coaxial dual-rotor system has two sets of uniform reverse rotation which has the same principle, parallel decomposition of symmetry. (2) alternate decomposition, multiple sub-principles cycle according to certain alternation. For example, compared with single cylinder mud pump, mud pump cylinder alternates in two cylinders, using the same alternate symmetry decomposition principle. (3) the choice of decomposition, the difference of sub-principle, at any time choosing a sub-principle, like aircraft and manual driving and automatic driving, is the principle of decomposition symmetry difference in the circumstance of choice .

Fifth, single and tandem symmetry, such as hydraulic system filter coexisting jam and breakdown. (4) filter series parallel decomposition symmetry scheme is to ensure that in any failure state oil can be normally filtered. Compared with the single filter it has series parallel decomposition symmetry.

\section{Two Design Principles and the Applications of the Principle Decomposition Symmetry}

First, decomposition principle of symmetrical components compatibility design: a mechanical system, rotation and translation of the space or the reverse principle are same and symmetric: mirror symmetry with multiple seeds also has the compatibility between the two, such as automobiles and a set of single direction tires of front space mirror symmetry, along the central axis tires in arbitrary plane mirror symmetrical. If the shape is different between the top left and both sides, that means the two tires can't transfer. If the mode shape is same between the top left and the both sides of the mirror, the mirrors on both sides of the tire are symmetric and these two tires can be interchangeable.

Second, the design rule of symmetry goals decomposition principle: in order to improve the performance and object-oriented efficiency of different properties, same and different application 
functions of the symmetry principles are decomposed in different scales. Performance as the objective function is same, the same principle can also be applied to the lines of symmetry decomposition that realized some of the same function. In most cases, it helps to improve product quality, improve the working efficiency, increase the complexity of system structure and the manufacturing cost.

Impedance composite muffler and double printers, respectively parallel decompose symmetry and improve the same performance of the principle by the various effect decomposition. As a single rotor turbofan engine, rotor synchronously driving the fan at a different speed, low pressure compressor and high pressure compressor, make the system performance difference, double rotor turbofan engine driven fan and the low-pressure compressor rotor rotating speed, high pressure compressor rotor high-speed drive, three turbofan engine rotor and rotor respectively to optimize speed-driven fan. Low pressure and high pressure compressors and the scale decomposition symmetry of the principle of the after-compared single rotor turbofan engine, improve the performance of the system and also the difficulty of the complex systems.

\section{Conclusion}

Symmetry exists widely in the function, principle and structure of the mechanical system and plays a very important role in technical, economic and social characteristics of the mechanical products. It realizes the product innovation to summarize the regularity knowledge of the application of mechanical symmetry, extract the design standards of mechanical symmetry, improve product performance, promote product innovation and improve the design efficiency. In the mechanical system principle decomposition symmetry, the innovation process of the existing product principle has been realized. Based on the principle decomposition symmetry, more innovative products can be invented, the inherent function and its performance of mechanical can be improved, thus, the application of the mechanical system principle decomposition symmetry in the future will be wider.

\section{Reference}

[1] Pei-en, Feng, Ling-bin, Zeng, Qing-ying, Qiu, Li-xin, Li. Working Mechanism and Its Application of Mechanical Principle Decomposition Symmetry[J]. Journal of Zhejiang University (Engineering Science), 2014, 02: 181-189+199.

[2] Ling-bin, Zeng. Research on Mechanical Function Symmetry and Principle Symmetry Concept and Its Application Knowledge System[D]. Zhejiang University, 2013.

[3] Yu, Liu. Mechanical Symmetry Knowledge Mining Method and Its Application in Mechanical Structure Rotational Symmetry[D]. Zhejiang University, 2012.

[4] Pei-en, Feng, Ling-bin, Zeng, Qing-ying, Qiu, Wei-ping, Liu. Research on Mechanical Function Symmetry Architecture and Its Application[J]. Journal of Mechanical Engineering, 2012, 11: 1-10.

[5] Zhi-yong, Ma. Research on Mechanical Symmetry Concept, Function and Acquisition of Application Knowledge[D]. Zhejiang University, 2010. 